\title{
TOLERANCE-CONTROL SOLUTIONS TO INTERVAL LINEAR EQUATIONS
}

\author{
LI TIAN ${ }^{1}$, WEI LI ${ }^{1}$, QIN WANG ${ }^{2}$ \\ ${ }^{1}$ Institute of Operational Research \& Cybernetics, Hangzhou Dianzi University, Hangzhou, 310018, PR China \\ ${ }^{2}$ College of Science, China Jiliang University, 310018, PR China \\ E-MAIL: t110081029@yahoo.cn,weilihz@126.com,wq@cjlu.edu.cn
}

\begin{abstract}
:
In this paper, we discuss the solution of interval linear equations from the point of view of the vector components. Tolerance solutions, control solutions, algebraic solutions and localized solutions are defined based on the relationship of two vectors as a whole, this paper detailed considerate the relationship of components between the two vectors. A new type of solutions, tolerance-control solutions, to interval linear equations are proposed, and the characteristics of the new solutions are described, which is similar to the well-know Oettli-Prager inequality.
\end{abstract}

\section{Keywords:}

Interval linear equations; Tolerance solutions; Control solutions; Algebraic solutions; L-localized solutions; R-localized solutions; Tolerance-control solutions.

\section{Introduction}

In recent years, a lot of uncertainty problems have been proposed,interval often be used as a special method to deal with these problems[2]. Owing to this,many scholars [3,6,8,10,11] have made some results about the methods for the interval linear equations $\mathbf{A} x=\mathbf{b}$, especially the solutions to $\mathbf{A} x=\mathbf{b}$. The united solutions were introduced from different viewpoint [3,5], and M.Hladík [9], S.P.Shary [14], J.Rohn [16] give some methods to bound the solution set. Some research has initially applied to practical problems, such as bounding roundoff errors in scientific computing.

As is know to all of us, tolerance solutions are defined based on $\mathbf{A} x \subseteq \mathbf{b}$, control solutions are defined based on $\mathbf{A} x \supseteq \mathbf{b}$, algebraic solutions based on $\mathbf{A} x=\mathbf{b}$. These solutions focus on the relationship of $\mathbf{A} x$ and $\mathbf{b}$, in this paper, the relationship of $(\mathbf{A} x)_{i}$ and $\mathbf{b}_{i}$ are considered. And this paper divided the solution set of weak solution set. Through the division, we see that tolerance solutions, control solutions and algebraic solutions are weak solutions with special, neat form. This paper introduced another solution of $\mathbf{A} x=\mathbf{b}$, based on the relationship of $(\mathbf{A} x)_{i}$ and $\mathbf{b}_{i}$. And the characterizations of the new solution is given, which is similar to Oettli-Prager inequality.

\section{Preliminaries}

Throughout this paper, we follow the definitions and notations given in [20].

$\underline{X}, \bar{X} \in R, X=[\underline{X}, \bar{X}]$ is called an interval number, where $\underline{X}$ and $\bar{X}$ are referred to as its lower and upper bounds. Similarly, if $\underline{A}, \bar{A} \in R^{m \times n}$, and $\underline{A} \leq \bar{A}, \mathbf{A}=[\underline{A}, \bar{A}]=\{A \mid \underline{A} \leq A \leq \bar{A}\}$ is called an interval matrix, and $\underline{A}, \bar{A}$ are referred to as its lower and upper bounds. If $\underline{A}=\left(a_{i j}\right), \bar{A}=\left(\overline{a_{i j}}\right)$, then we have

$\mathbf{A}=\left\{A=\left(a_{i j}\right) \mid a_{i j} \leq a_{i j} \leq \overline{a_{i j}}, i=1, \cdots, m, j=1 \cdots, n\right\}$.

The center and radius matrices are denoted by $A_{c}$ and $A_{\Delta}$

$$
A_{c}=\frac{1}{2}(\underline{A}+\bar{A}), A_{\Delta}=\frac{1}{2}(\bar{A}-\underline{A}) \text {. }
$$

An interval vector $\mathbf{b}$ can be defined as an one-column interval matrix. Similarly,

$$
\mathbf{b}=\{b \mid \underline{b} \leq b \leq \bar{b}\}, \underline{b}, \bar{b} \in R^{m} .
$$

The center and radius vectors are defined as

$$
b_{c}=\frac{1}{2}(\underline{b}+\bar{b}), b_{\Delta}=\frac{1}{2}(\bar{b}-\underline{b}) .
$$

Let

$$
Y_{m}=\left\{y \in R^{m}|| y \mid=e\right\},
$$

where $e=(1, \cdots, 1)^{T}$. For each $x \in R^{n}$,we define its diagonal vector as

$$
(\operatorname{sgn} x)_{i}= \begin{cases}1, & x_{i} \geq 0 \\ -1, & x_{i}<0\end{cases}
$$




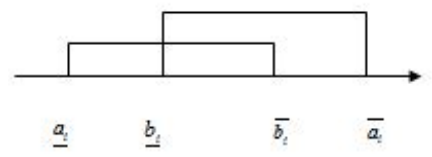

(1)

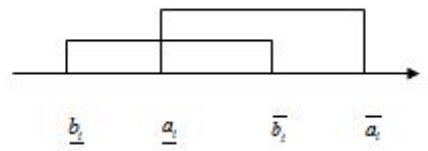

(3)

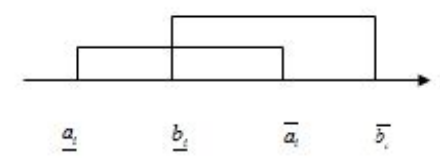

(2)

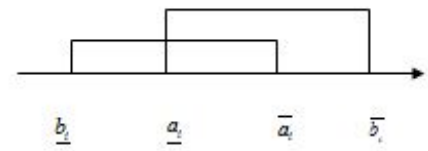

(4)
Figure 1.

For a given vector $y \in R^{m}$,we define

$$
T_{y}=\operatorname{diag}\left(y_{1}, \cdots, y_{m}\right)=\left(\begin{array}{cccc}
y_{1} & 0 & \cdots & 0 \\
0 & y_{2} & \cdots & 0 \\
\vdots & \vdots & \ddots & \vdots \\
0 & 0 & \cdots & y_{m}
\end{array}\right) .
$$

Then we can write $|x|=T_{z} x$, where $z=\operatorname{sgn} x$.

For a given matrix $\mathbf{A}=\left[A_{c}-A_{\Delta}, A_{c}+A_{\Delta}\right]$ and $y \in Y_{m}, z \in$ $Y_{n}$, we introduce the following matrices:

$$
A_{y z}=A_{c}-T_{y} A_{\Delta} T_{z}
$$

Lemma 1 For two interval vectors $\mathbf{a}=\left(\mathbf{a}_{1}, \cdots, \mathbf{a}_{n}\right)^{T}, \mathbf{b}=$ $\left(\mathbf{b}_{1}, \cdots, \mathbf{b}_{n}\right)^{T}, \mathbf{a} \cap \mathbf{b} \neq \phi$ is equivalent to $\underline{a} \leq \bar{b}, \underline{b} \leq \bar{a}$.

Proof. If $\mathbf{a} \cap \mathbf{b} \neq \phi$, then $\exists c \in R^{n}, c \in \mathbf{a}, c \in \mathbf{b}$, which means

$$
\underline{a} \leq c \leq \bar{a}, \underline{b} \leq c \leq \bar{b}
$$

We have

$$
\underline{a} \leq c \leq \bar{b}, \underline{b} \leq c \leq \bar{a}
$$

so

$$
\underline{a} \leq \bar{b}, \underline{b} \leq \bar{a}
$$

Conversely, if $\underline{a} \leq \bar{b}, \underline{b} \leq \bar{a}$, then $\underline{a_{i}} \leq \overline{b_{i}}, \underline{b_{i}} \leq \overline{a_{i}}$. We have the following four cases:

(1) $\underline{a_{i}} \leq \underline{b_{i}} \leq \overline{b_{i}} \leq \overline{a_{i}}$, (2) $a_{i} \leq \underline{b_{i}} \leq \overline{a_{i}} \leq \overline{b_{i}}$,

(3) $\overline{b_{i}} \leq \overline{a_{i}} \leq \overline{b_{i}} \leq \overline{a_{i}}$, (4) $\overline{b_{i}} \leq \overline{a_{i}} \leq \overline{a_{i}} \leq \overline{b_{i}}$.

Figures $\overline{1}$ is more clearer to explain the four inequality.

For case (1), $\left(b_{c}\right)_{i} \in \mathbf{a}_{i} \cap \mathbf{b}_{i}$; for case (2), $\frac{b_{i}+\overline{a_{i}}}{2} \in \mathbf{a}_{i} \cap \mathbf{b}_{i}$; for case (3), $\frac{a_{i}+\overline{b_{i}}}{2} \in \mathbf{a}_{i} \cap \mathbf{b}_{i}$; for case (4), $\left(b_{c}\right)_{i} \in \mathbf{a}_{i} \cap \mathbf{b}_{i}$. No matter what case, one of the four numbers $\left(b_{c}\right)_{i}, \frac{b_{i}+\overline{a_{i}}}{2}, \frac{a_{i}+\overline{b_{i}}}{2},\left(b_{c}\right)_{i}$ belongs to $\mathbf{a}_{i} \cap \mathbf{b}_{i}$, we denote it with $k_{i}(i=1, \cdots, n)$. so we have $k_{i} \in \mathbf{a}_{i} \cap \mathbf{b}_{i}$, which implies $k=\left(k_{1}, \cdots, k_{n}\right)^{T} \in \mathbf{a} \cap \mathbf{b}$.

For two interval vectors $\mathbf{a} \cap \mathbf{b} \neq \phi$, i.e. $\underline{a} \leq \bar{b}, \underline{b} \leq \bar{a}$. if we consider the sequence relationship of their components, we can get some results. Let

$$
\begin{gathered}
U=\{1,2, \cdots, n\} \\
\Omega_{1}=\left\{i \mid \underline{a_{i}}<\underline{b_{i}}\right\}, \Omega_{2}=\left\{i \mid \underline{a_{i}}=\underline{b_{i}}\right\}, \Omega_{3}=\left\{i \mid \underline{a_{i}}>\underline{b_{i}}\right\} \\
\Gamma_{1}=\left\{j \mid \overline{b_{j}}<\overline{a_{j}}\right\}, \Gamma_{2}=\left\{j \mid \overline{b_{j}}=\overline{a_{j}}\right\}, \Gamma_{3}=\left\{j \mid \overline{b_{j}}>\overline{a_{j}}\right\}
\end{gathered}
$$

We can see that for every subscript $i$, there are $c_{3}^{1} c_{3}^{1}$ cases. So if the weak solutions are divided by component, there are $9^{n}$ cases.

Lemma 2 If interval number $\mathbf{a}=[\underline{a}, \bar{a}], x \in R$, then $(\mathbf{a} x)_{c}=$ $a_{c} x$.

Proof. (i) If $x \geq 0$, we have $\underline{a x} \leq \bar{a} x$, which gives $\mathbf{a} x=$ $[\underline{a} x, \bar{a} x],(\mathbf{a} x)_{c}=\frac{\underline{a} x+\bar{a} x}{2}=\frac{(\underline{a}+\bar{a}) x}{2}=a_{c} x$. (ii) If $x<0$, then $\mathbf{a} x=[\bar{a} x, \underline{a} x]$, we have $(\mathbf{a} x)_{c}=\frac{\bar{a} x+\underline{a} x}{2}=\frac{(\bar{a}+\underline{a}) x}{2}=a_{c} x$. We can conclude that $(\mathbf{a} x)_{c}=a_{c} x$.

Lemma 3 For interval vector $\mathbf{a}, x \in R$, we have $(\mathbf{a} x)_{\Delta}=$ $a_{\Delta}|x|$.

Proof. When $x \geq 0$, we have $\mathbf{a} x=[\underline{a} x, \bar{a} x],(\mathbf{a} x)_{\Delta}=$ $\frac{\bar{a} x-\underline{a} x}{2}=a_{\Delta}|x|$; When $x<0$, we have $(\mathbf{a} x)_{\Delta}=\frac{a x-\bar{a} x}{2}=$ $-a_{\Delta} x=a_{\Delta}|x|$. So we conclude that $(\mathbf{a} x)_{\Delta}=a_{\Delta}|x|$.

Lemma 4 Interval matrix $\mathbf{A}, x \in R^{n}$, then there holds $\mathbf{A} x=\left[A_{c} x-A_{\Delta}|x|, A_{c} x+A_{\Delta}|x|\right]=\left[\mathbf{A}_{e z} x, \mathbf{A}_{-e z} x\right]$, where $z=\operatorname{sgn} x$.

Proof. Firstly, we prove The first equation, $\underline{\mathbf{A} x}=(\mathbf{A} x)_{c}-$ $(\mathbf{A} x)_{\Delta}=A_{c} x-A_{\Delta}|x|$. Similarly, $\overline{\mathbf{A} x}=\left(\overline{\mathbf{A} x)_{c}}+(\mathbf{A} x)_{\Delta}=\right.$ $A_{c} x+A_{\Delta}|x|$.

Now we prove the second equation, for $|x|=T_{z} x$, we have $A_{c} x-A_{\Delta}|x|=A_{c} x-A_{\Delta} T_{z} x=\left(A_{c}-A_{\Delta} T_{z}\right) x=A_{e z} x$. Similarly, $A_{c} x+A_{\Delta}|x|=A_{c} x+A_{\Delta} T_{z} x=\left(A_{c}+A_{\Delta} T_{z}\right) x=$ $A_{-e z} x$.

we have $(\mathbf{A} x)_{i}=\left[(\underline{\mathbf{A} x})_{i},(\overline{\mathbf{A} x})_{i}\right]=\left[\left(A_{c} x\right)_{i}-\right.$ $\left.\left(A_{\Delta}|x|\right)_{i},\left(A_{c} x\right)_{i}+\left(A_{\Delta}|x|\right)_{i}\right]=\left[\left(A_{e z} x\right)_{i},\left(A_{-e z} x\right)_{i}\right]$

\section{Division of the weak solution}

$x$ is a weak solution of interval linear equations $\mathbf{A} x=\mathbf{b}$ is equivalent to $\mathbf{A} x \cap \mathbf{b} \neq \phi$. The set of weak solution is

$$
\sum_{\exists \exists}(\mathbf{A}, \mathbf{b})=\left\{x \in R^{n} \mid \mathbf{A} x \cap \mathbf{b}\right\} \neq \phi
$$

If $x$ is a weak solution of $\mathbf{A} x=\mathbf{b}$, then according to lemma 1 , there holds $\underline{\mathbf{A} x} \leq \bar{b}, \underline{b} \leq \overline{\mathbf{A} x}$, that is $A_{e z} x \leq \bar{b}, \underline{b} \leq A_{-e z} x$. For interval linear equations $\mathbf{A} x=\mathbf{b}$, the set of weak solution has the maximum range. If we consider based on components 
of vector, the set of weak solution can be divided into many parts. Let

$U=\{1, \cdots, m\}$,

$\Omega_{1}=\left\{i \mid(\mathbf{A} x)_{i}<\underline{b_{i}}\right\}, \Omega_{2}=\left\{i \mid \underline{(\mathbf{A} x)_{i}}=\underline{b_{i}}\right\}$,

$\Omega_{3}=\left\{i \mid \overline{(\mathbf{A} x)_{i}}>\underline{b_{i}}\right\}$.

$\Gamma_{1}=\left\{j \mid \overline{\overline{b_{j}}<\overline{(\mathbf{A} x)_{j}}}\right\}, \Gamma_{2}=\left\{j \mid \overline{b_{j}}=\overline{(\mathbf{A} x)_{j}}\right\}$,

$\Gamma_{3}=\left\{j \mid \overline{b_{j}}>\overline{(\mathbf{A} x)_{j}}\right\}$.

So we have:

(1) $\Omega_{1} \cup \Omega_{2}=U, \Gamma_{1} \cup \Gamma_{2}=U \Leftrightarrow \underline{\mathbf{A} x} \leq \underline{b} \leq \bar{b} \leq \overline{\mathbf{A} x} \Leftrightarrow \mathbf{b} \subseteq$ A $x$, which implies $x$ is a control solution.

(2) $\Omega_{2} \cup \Omega_{3}=U, \Gamma_{2} \cup \Gamma_{3}=U \Leftrightarrow \underline{b} \leq \underline{\mathbf{A} x} \leq \overline{\mathbf{A} x} \leq \bar{b} \Leftrightarrow$ $\mathbf{A} x \subseteq \mathbf{b}$, which implies $x$ is a tolerance solution.

(3) $\Omega_{2}=U, \Gamma_{2}=U \Leftrightarrow \underline{\mathbf{A} x}=\underline{b} \leq \bar{b}=\overline{\mathbf{A} x} \Leftrightarrow \mathbf{A} x=\mathbf{b}$, which implies $x$ is an algebraic solution.

In addition, if $\mathbf{A} x \leq \bar{b}, \underline{b} \leq \overline{\mathbf{A} x}$, we have

$\Omega_{1} \cup \Omega_{2}=\overline{U, \Gamma_{2}} \cup \Gamma_{3}=U \Leftrightarrow \underline{\mathbf{A} x} \leq \underline{b} \leq \overline{\mathbf{A} x} \leq \bar{b}$, which implies $x$ is a L-localized solution.

$\Omega_{2} \cup \Omega_{3}=U, \Gamma_{1} \cup \Gamma_{2}=U \Leftrightarrow \underline{b} \leq \underline{\mathbf{A} x} \leq \bar{b} \leq \overline{\mathbf{A} x}$, which implies $x$ is a R-localized solution.

These are just some special form of weak solutions, there are also a lot of weak solution which has not be discussed.

For example, $x=(1,3)^{T}$ is an weak solution of

$$
\left(\begin{array}{cc}
{[1,2]} & {[4,6]} \\
{[-1,3]} & {[2,5]}
\end{array}\right) x=\left(\begin{array}{c}
{[12,21]} \\
{[6,20]}
\end{array}\right)
$$

We can see that

$$
\begin{gathered}
([1,2] \quad[4,6]) x=[13,20] \subseteq[12,21] \\
([-1,3] \quad[2,5]) x=[5,18] \cap[6,20] \neq \phi .
\end{gathered}
$$

$x$ isn't a tolerance solution, nor is it a control solution, it is just a weak solution with $\Omega_{1}=\{2\}, \Omega_{2}=\phi, \Omega_{3}=\{1\}, \Gamma_{1}=$ $\phi, \Gamma_{2}=\phi, \Gamma_{3}=\{1,2\}$.

For another example, $x=(-1,2)^{T}$ is a weak solution of

$$
\left(\begin{array}{cc}
{[-1,3]} & {[2,5]} \\
{[1,2]} & {[-2,1]}
\end{array}\right) x=\left(\begin{array}{c}
{[0,11]} \\
{[-4,0]}
\end{array}\right)
$$

We can see that

$$
\begin{gathered}
([-1,3] \quad[2,5]) x=[1,11] \subseteq[0,11] \\
([1,2] \quad[-2,1]) x=[-6,1] \supseteq[-4,0]
\end{gathered}
$$

$x$ is a weak solution with $\Omega_{1}=\{2\}, \Omega_{2}=\phi, \Omega_{3}=\{1\}$, $\Gamma_{1}=\{2\}, \Gamma_{2}=\{1\}, \Gamma_{3}=\phi$.

Based on the examples, we know that tolerance solutions, control solutions and algebraic solutions are weak solutions of special type. Different combinations of $\Omega_{1}, \Omega_{2}, \Omega_{3}$ and $\Gamma_{1}, \Gamma_{2}, \Gamma_{3}$

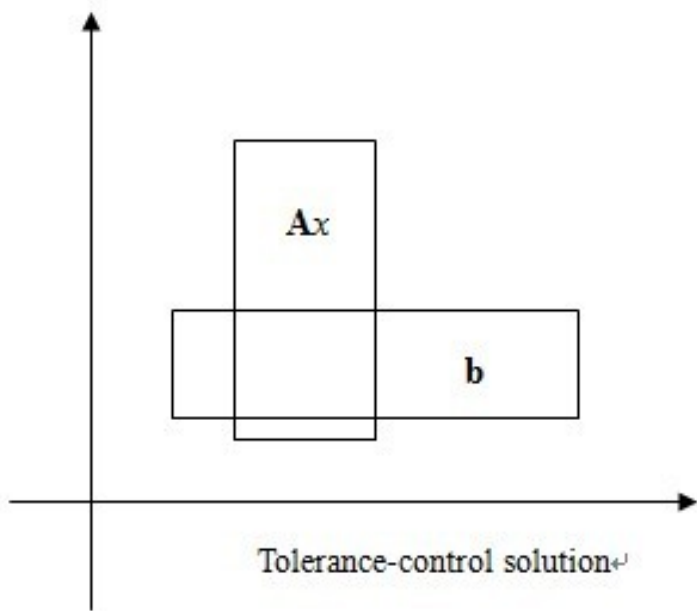

Figure 2.

make up difference types of weak solutions. Next, we introduce another type of weak solution.

\section{The definition of tolerance-control solution to in- terval linear equations}

For interval linear equations $\mathbf{A} x=\mathbf{b}$, let $T=\left\{i \mid(\mathbf{A} x)_{i} \subseteq\right.$ $\left.\mathbf{b}_{i}\right\}, C=\left\{i \mid(\mathbf{A} x)_{i} \supseteq \mathbf{b}_{i}\right\}, U=\{1, \cdots, m\}$.

A vector $x \in R^{n}$ is called a tolerance-control solution of interval linear equations if there holds $T \cup C=U$. Especially, if $T=U, x$ is a tolerance solution; if $C=U, x$ is a control solution. We can see the differences between tolerance solution, control solution, L-localized solution, R-localized solution and the new solution, tolerance-control solution in the two-dimensional case(see figure 2 and figure 3 ).

The introduction of tolerance-control solution make solutions of interval linear equations more perfect, what's more, it has important significance and application value, take "black box" (see Figure4) for example. $x \in R^{n}$ is the input data, the input-output relationship is $y=A x$, where $A \in R^{m \times n}$, $A=\left(a_{i j}\right)$. Suppose that we don't know the precise data of the element $a_{i j}$, but we know it is within a interval $\mathbf{a}_{i j}$, i.e. $a_{i j} \in \mathbf{a}_{i j}$, which constitutes an interval matrix $\mathbf{A}=\left(\mathbf{a}_{i j}\right)$.

In this case, the "black box" has been transformed into interval linear equations. Sergey P.Shary use this model to introduce the tolerance solution and the control solution. For the required tolerance $\mathbf{b}$ and any values of the parameters $a_{i j} \in \mathbf{a}_{i j}$. if the input signals $x$ satisfies $\mathbf{A} x \subseteq \mathbf{b}, x$ is called a tolerance solu- 

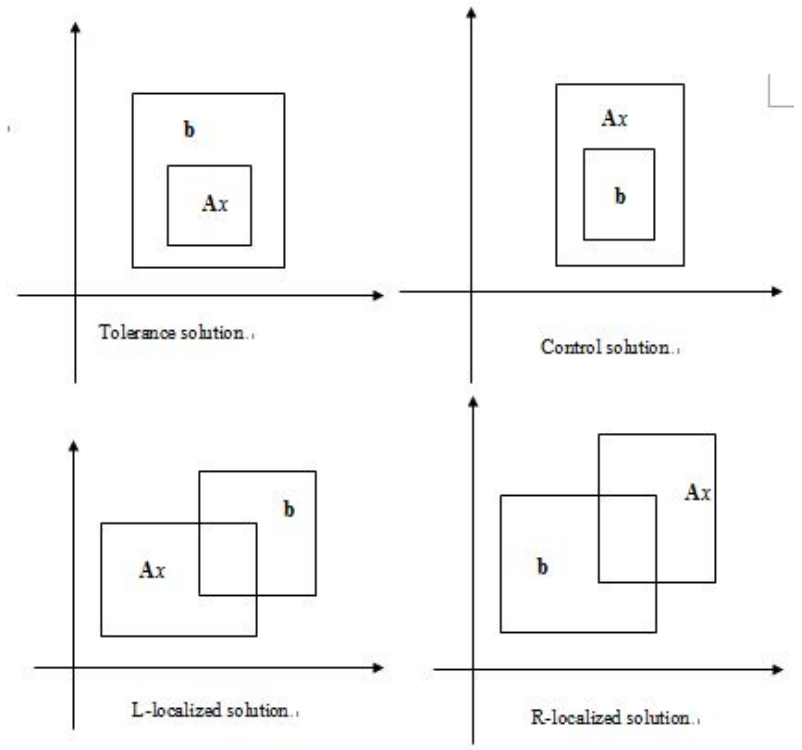

Figure 3.

tion. If the input signal $x$ satisfies $\mathbf{A} x \supseteq \mathbf{b}, x$ is called a control solution. But for a input signal $x$, if some components satisfy $(\mathbf{A} x)_{i} \subseteq \mathbf{b}_{i}$, the rest components satisfy $(\mathbf{A} x)_{i} \supseteq \mathbf{b}_{i}$, we call this $x$ is a tolerance-control solution.

\section{The characterizations of tolerance-control solu- tion of interval linear equations}

Theorem 1 A vector $x \in R^{n}$ is called a tolerance-control solution of interval linear equations $\mathbf{A} x=\mathbf{b}$ if and only if there holds

$$
\begin{array}{r}
\left|\left(A_{c} x\right)_{i}-\left(b_{c}\right)_{i}\right| \leq-\left(A_{\Delta}|x|\right)_{i}+\left(b_{\Delta}\right)_{i}, \quad i \in T \\
\left|\left(A_{c} x\right)_{i}-\left(b_{c}\right)_{i}\right| \leq\left(A_{\Delta}|x|\right)_{i}-\left(b_{\Delta}\right)_{i}, \quad i \in C
\end{array}
$$

where $T \cup C=U$.

Proof. If $x$ is a tolerance-control solution, there holds $\exists i \in T$, satisfies $(\mathbf{A} x)_{i} \subseteq \mathbf{b}_{i} . \exists i \in C$ satisfies $(\mathbf{A} x)_{i} \supseteq \mathbf{b}_{i}$. We have

$$
(\mathbf{A} x)_{i}=\left[\left(A_{c} x\right)_{i}-\left(A_{\Delta}|x|\right)_{i},\left(A_{c} x\right)_{i}+\left(A_{\Delta}|x|\right)_{i}\right] .
$$

$(\mathbf{A} x)_{i} \subseteq \mathbf{b}_{i}(i \in T)$ implies

$$
\underline{b_{i}} \leq \underline{(\mathbf{A} x)_{i}} \leq \overline{(\mathbf{A} x)_{i}} \leq \overline{b_{i}}(i \in T),
$$

i.e.

$\left(b_{c}\right)_{i}-\left(b_{\Delta}\right)_{i} \leq\left(A_{c} x\right)_{i}-\left(A_{\Delta}|x|\right)_{i} \leq\left(A_{c} x\right)_{i}+\left(A_{\Delta}|x|\right)_{i} \leq\left(b_{c}\right)_{i}+\left(b_{\Delta}\right)_{i} \quad\left(A_{e z} x\right)_{i} \leq \underline{b_{i}},-\left(A_{-e z} x\right)_{i} \leq-\overline{b_{i}},(i \in C)$.

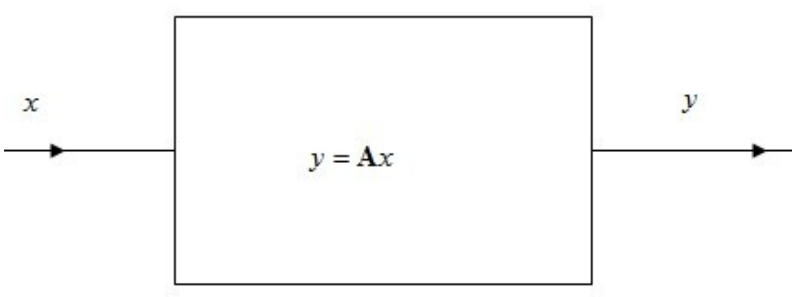

Figure 4.

where $(i \in T)$. which implies

$$
\left|\left(A_{c} x\right)_{i}-\left(b_{c}\right)_{i}\right| \leq-\left(A_{\Delta}|x|\right)_{i}+\left(b_{\Delta}\right)_{i},(i \in T) .
$$

Similarly, $(\mathbf{A} x)_{i} \supseteq \mathbf{b}_{i},(i \in C)$ can deduced

$$
\left|\left(A_{c} x\right)_{i}-\left(b_{c}\right)_{i}\right| \leq\left(A_{\Delta}|x|\right)_{i}-\left(b_{\Delta}\right)_{i},(i \in C) .
$$

we also have

$$
T \cup C=U
$$

Conversely, if there holds

$$
\left|\left(A_{c} x\right)_{i}-\left(b_{c}\right)_{i}\right| \leq-\left(A_{\Delta}|x|\right)_{i}+\left(b_{\Delta}\right)_{i},(i \in T)
$$

then it can deduced that $(\mathbf{A} x)_{i} \subseteq \mathbf{b}_{i},(i \in T)$.

Similarly,

$$
\left|\left(A_{c} x\right)_{i}-\left(b_{c}\right)_{i}\right| \leq\left(A_{\Delta}|x|\right)_{i}-\left(b_{\Delta}\right)_{i},(i \in C)
$$

can deduced that $(\mathbf{A} x)_{i} \supseteq \mathbf{b}_{i},(i \in C)$. In addition,

$$
T \cup C=U,
$$

which gives that $x$ is a tolerance-control solution. $\square$

Theorem 2 A vector $x \in R^{n}$ is called a tolerance-control solution of interval linear equations $\mathbf{A} x=\mathbf{b}$ if and only if there holds

$$
\begin{gathered}
-\left(A_{e z} x\right)_{i} \leq-\underline{b_{i}}, \quad i \in T \\
\left(A_{-e z} x\right)_{i} \leq \overline{b_{i}}, \quad i \in T \\
\left(A_{e z} x\right)_{i} \leq \underline{b_{i}}, \quad i \in C \\
-\left(A_{-e z} x\right)_{i} \leq-\overline{b_{i}}, \quad i \in C
\end{gathered}
$$

where $T \cup C=U$.

Proof. According to lemma 6, there holds $\underline{\mathbf{A} x}=A_{e z} x, \overline{\mathbf{A} x}=$ $A_{-e z} x$, then we have

$$
\underline{(\mathbf{A} x)_{i}}=\left(A_{e z} x\right)_{i}, \overline{(\mathbf{A} x)_{i}}=\left(A_{-e z} x\right)_{i},(i \in T)
$$

So $(\mathbf{A} x)_{i} \subseteq \mathbf{b}_{i},(i \in T)$ is equivalent to

$$
-\left(A_{e z} x\right)_{i} \leq-\underline{b_{i}},\left(A_{-e z} x\right)_{i} \leq \overline{b_{i}},(i \in T)
$$

$(\mathbf{A} x)_{i} \supseteq \mathbf{b}_{i},(i \in C)$ is equivalent to$$
\left(A_{e z} x\right)_{i} \leq \underline{b_{i}},-\left(A_{-e z} x\right)_{i} \leq-b_{i},(i \in C)
$$ 


\section{Conclusions}

In this paper, we have divided weak solutions of interval linear equations based on vector components, we know that tolerance solutions, control solutions and algebraic solutions are weak solutions with special form. In addition, there are many other types of weak solutions we haven't discussed. Another important solution, the tolerance-control solution is introduced, the paper described the characterizations of the new solutions.

\section{Acknowledgements}

This project was supported by the National Natural Science Foundation of China (Grant No. 61003194,11171316)and Zhejiang province university students' innovative and undertaking incubator program (R407061).

\section{References}

[1] Wei Li,Huping Wang,Qing Wang.Localized solutions to interval linear equations.journal of computational and applied mathematics, 2012,238(15):29-38.

[2] Baoding Liu, Theory and practice of uncertain programming, third ed., Springer, Berlin, 2009.

[3] G. Alefeld, J. Herzberger, Introduction to Interval Computations, Academic Press, NewYork, 1983.

[4] A. Neumaier, Interval Methods for Systems of Equations, Cambridge University Press, Cambridge, 1990.

[5] J. Rohn, Systems of linear interval equations, Linear Algebra Appl. 126 (1989) 39-78.

[6] G. Alefeld, V. Kreinovich, G. Mayer, On the solution sets of particular classes of linear interval systems, J. Comput. Appl. Math. 152 (2003) 1-15.

[7] M. Hladík, Interval Linear Programming: A Survey, In: Zoltan Adam Mann Edits, Linear Programming New Frontiers. Nova Science Publishers Inc., 2011.

[8] M. Hladík, Optimal value bounds in nonlinear programming with interval data, TOP 19 (1) (2011) 93-106. doi:10.1007/s11750-009-0099-y.

[9] M. Hladík, Description of symmetric and skewsymmetric solution set, SIAM J. Matrix Anal. Appl. 30 (2)(2008) 509-521.

[10] M. Hladík, Optimal value range in interval linear programming, Fuzzy Optim. Decis. Mak. 8(3) (2009) 283294.

[11] M. Hladík, Additive and multiplicative tolerance in multiobjective linear programming, Oper. Res. Lett. 36(3) (2008) 393-396.
[12] M. Hladík, Multiparametric linear programming: support set and optimal partition invariancy, Technical Report KAMDIMATIA Series(2007-815), Department of Applied Mathematics, Prague, 2007.

[13] M. Hladłk, Tolerance analysis in linear programming, Technical Report KAMDIMATIA Series (2008-901), Department of Applied Mathematics, Prague, 2008.

[14] Aharon Ben-Tal, A.Goryashko, E.Guslitzer, Arkadi Nemirovski, Adjustable robust solutions of uncertain linear programs, Math. Program. 99 (2004), no.2.

[15] E. Nuding and J. Wilhelm, Über Gleichungen und über Lösungen, Zeitschrift für Angewandte Mathematik und Mechanik, 52 (1972) 188-190.

[16] J. Rohn, Input-output planning with inexact data, Freiburger Intervall-Berichte 78/9, Albert-Ludwigs- Universität, Freiburg, 1978.

[17] Wei Li, Xiaoli Tian, Numerical solution method for general interval quadratic programming, Applied Mathematics and Computation, 202 (2008) 589-595.

[18] Wei Li, Xiaoli Tian, Fault detection in discrete dynamic systems with uncertainty based on interval optimization, Mathematical modelling and analysis, 16(4) (2011) 549557.

[19] A. Neumaier, Interval Methods for Systems of Equations, Cambridge University Press, Cambridge, 1990.

[20] M. Fiedler, J. Nedoma, J. Ramik, J. Rohn, K. Zimmermann, Linear optimization problems within exact data, Springer, New York, 2006.

[21] A. Neumaier, Tolerance analysis with interval arithmetic, Freiburger Intervall-Berichte 86(9) (1986) 5-19.

[22] B. Kelling, D. Oelschliagel, Zur Losung von linearen Toleranzproblemen, Wiss. Z. TH Leuna-Merseburg 33(1) (1991) 121-131.

[23] S. P. Shary, Solving the interval linear tolerance problem, Math. Comput Simulation 39 (1995) 53-85.

[24] S. P. Shary, Controllable solution set to interval static systems, Math. Comput Simulation 86 (1997) 185-196. 